\title{
Obliterative le fort's colpocleisis for acute kidney injury caused by pelvic organ prolapse in elderly woman
}

\author{
Rohini E. ${ }^{1 *}$, Hiremath P. B. ${ }^{1}$, Urmi Sanyal ${ }^{1}$, Indu N. R. ${ }^{1}$, Shilpa Ghosh ${ }^{1}$, Reshma Hiremath ${ }^{2}$
}

${ }^{1}$ Department of Obstetrics and Gynecology, SVMCH and RC, Ariyur, Puducherry, India

${ }^{2}$ Department of Obstetrics and Gynaecology, Mapusa, Goa, India

Received: 12 July 2019

Accepted: 10 September 2019

*Correspondence:

Dr. Rohini E.,

E-mail: roh8846@gmail.com

Copyright: (c) the author(s), publisher and licensee Medip Academy. This is an open-access article distributed under the terms of the Creative Commons Attribution Non-Commercial License, which permits unrestricted non-commercial use, distribution, and reproduction in any medium, provided the original work is properly cited.

\begin{abstract}
Pelvic organ prolapse (POP) is the descent of pelvic organs through the vagina, which sometimes causes hydronephrosis. Here authors report a case of an eighty five year old woman with a fourth degree uterine prolapse with obstructive uropathy. She was treated with a conservative surgery Le Fort's colpocleisis. Following which the patient's renal functions and symptoms improved. Hence authors conclude that colpocleisis can be considered as the option for elderly women who have completed the family with no desire to preserve the sexual function especially in women with co morbities where pelvic reconstructive surgeries pose a challenge.
\end{abstract}

Keywords: Colpoclesis, Conservative surgery, Obstructive uropathy, Pelvic organ prolapse

\section{INTRODUCTION}

Colpocleisis is an obliterative surgery for women with uterovaginal or vaginal vault prolapse, who do not intend to preserve vaginal function for sexual function. The procedure was first described by Leon Le Fort in 1877.

Decision of the type of treatment for genital prolapse in elderly women is a challenging situation. ${ }^{1}$ Pelvic reconstructive surgery is especially challenging in elderly and advanced age women with high stage POP as most of these women have comorbidities such as cardiovascular diseases, chronic pulmonary diseases, impaired renal function, diabetes mellitus or malignancy, which increases the incidence of adverse outcomes during and after surgical intervention. $^{2}$

\section{CASE REPORT}

An eighty five year old, post menopausal para 4 living 4 woman visited the department of gynaecology at Sri
Venkateshwaraa medical college, Puducherry with complaints of mass descending per vaginum since four years, acute retention of urine for the past 3 days and associated with lower abdominal pain. The mass was insidious in onset and progressive in nature. She had no past medical illness or any past history of surgery. On general physical examination, she was poorly built and nourished. On per abdomen examination a pelvic mass was palpable up to a uterine size of 24 weeks, tender and cystic in consistency. On local examination a fourth degree uterovaginal prolapse with edema was noted. Blood investigations done revealed $\mathrm{aHb}$ of $9.5 \%$, renal function tests being deranged. Serum creatinine being $3 \mathrm{mg} / \mathrm{dl}$ and serum urea $50 \mathrm{mg} / \mathrm{dl}$. Ultrasound abdomen revealed bilateral severe hydroureteronephrosis and a large ovarian cyst of $11.5 \mathrm{X} 9.5 \mathrm{~cm}$ with no features of malignancy.

The prolapse was reduced after douching. A ring pessary placed. 2 days later the serum creatinine had dropped to $1.9 \mathrm{mg} / \mathrm{dl}$ and serum urea to $42 \mathrm{mg} / \mathrm{dl}$. 
Considering the old age and the symptoms, with the aim of giving a better quality of life and avoiding the morbidity associated with extensive pelvic surgery, authors did a conservative surgery, Colpocleisis (Le Fort's) procedure after counselling the patient and her attenders. Hence the prolapse was reduced and the vagina was obliterated surgically. Post-operative period was uneventful. Urinary catheter was removed on post operative day 4 . She could pass urine without difficulty. She was discharged on day seven. Her Renal function test done on post op day 7 . Serum creatinine $1.8 \mathrm{mg} / \mathrm{dl}$ and serum urea $36 \mathrm{mg} / \mathrm{dl}$.

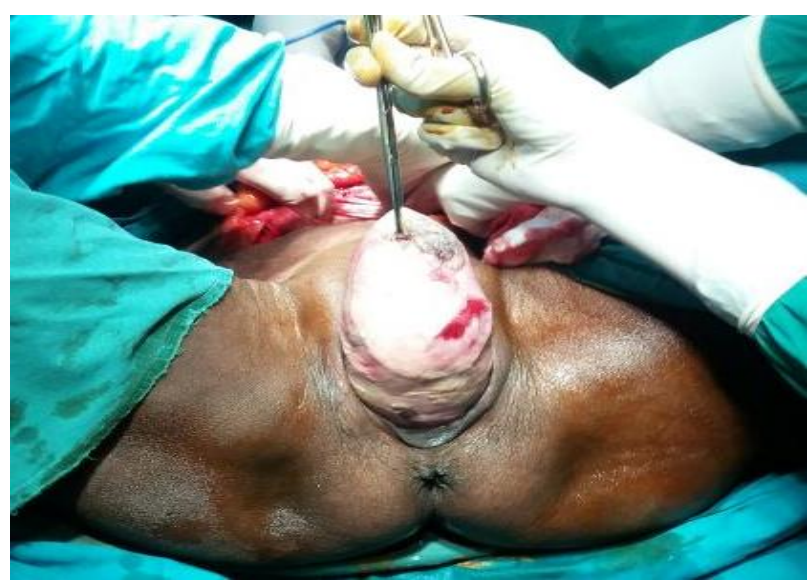

Figure 1: Procidentia just prior to the surgery.

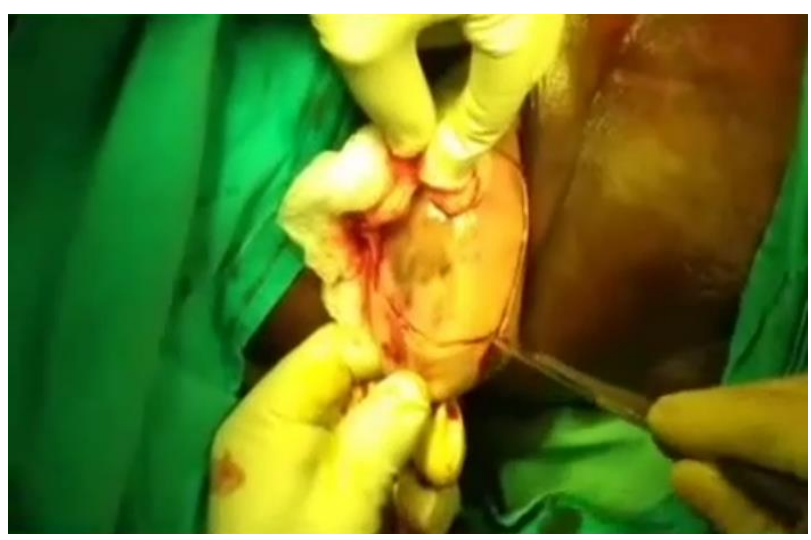

Figure 2: Incision on the anterior vaginal wall.

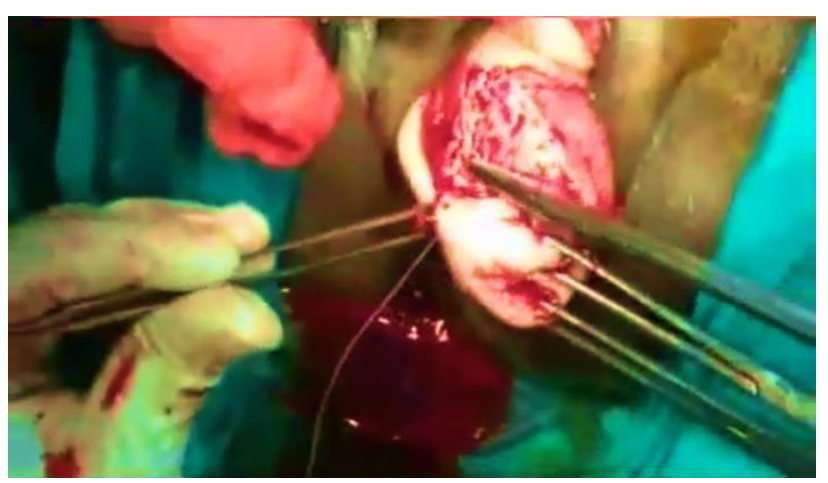

Figure 3: Dissection of the anterior vaginal wall.

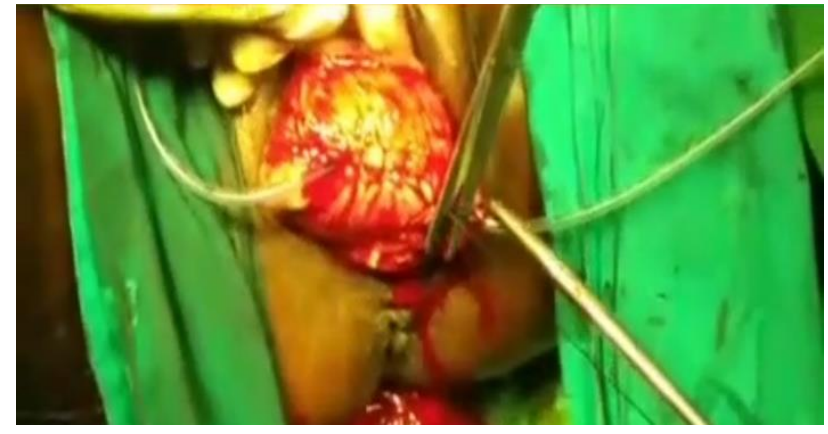

Figure 4: Sequential obliteration of the vagina.

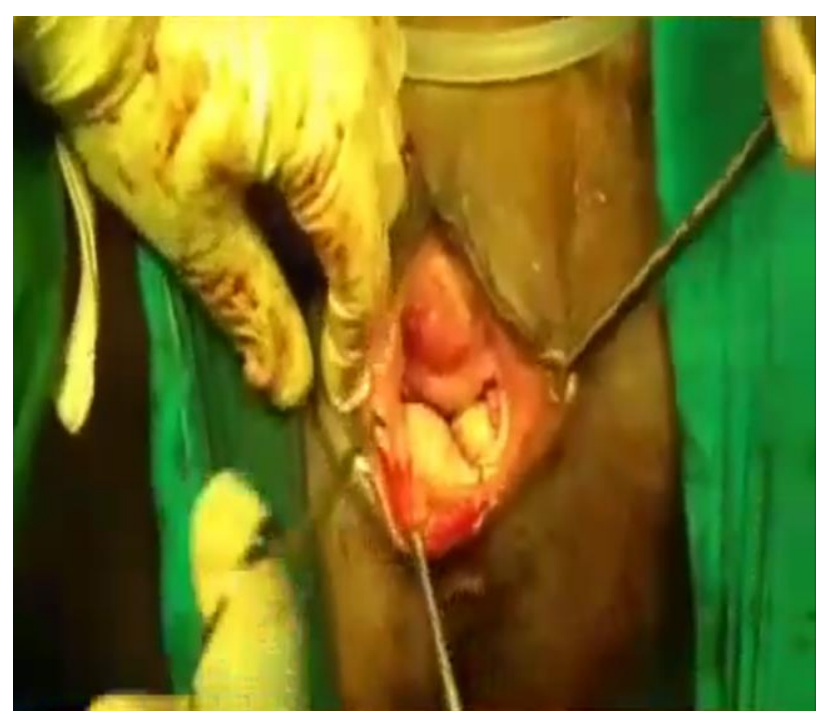

Figure 5: End result of the procedure.

\section{DISCUSSION}

Among the complications of POP hydronephrosis is one of the most serious one whichmay lead to renal dysfunction or urinary infection. ${ }^{3}$ The mechanism of hydronephrosis and hydroureter caused by POP may be explained by several hypotheses:

- Compression and obstruction of the ureters outside the bladder

- Kinking of the ureters caused by uterine artery or ligament

- Stasis in the cystocele caused by dysuria..$^{3-5}$

Vaginal ring pessaries have long been used for treatment of POP in elderly women. These provide support to related pelvic structures, and hence relieve pressure on the bladder and bowel. ${ }^{6,7}$ The complications of pessaries are vaginal discharge, foul smell, bleeding, constipation, and urinary tract infection and vesicovaginal and recto vaginalfistula. More commonly they cause a persistent discharge which may become blood stained due to superficial ulceration of the vaginal skin.

In a study conducted by Soo-Cheen $\mathrm{Ng}$ et al. Obliterative colpoclesis had a shorter operation time and less 
estimated blood loss compared with surgery involving vaginal hysterectomy with or without colporrhaphy. ${ }^{1}$

This procedure can be performed in advanced vaginal stump prolapse and also with preservation of the uterus. It does not necessitate deep and extensive tissue dissection as with trans-vaginal mesh insertion and also avoids an intra-abdominal approach as with sacral colpopexy. ${ }^{1}$

The advantage of Le Fort Colpocleisis is that it is the simplest and quickest of all procedures which can be used to correct prolapse. It can be performed using only light anaesthesia and will cause only minimal blood loss. ${ }^{2}$

The most common disadvantage of colpocleisis is that bleeding from the genital tract due to a carcinoma cannot be investigated properly, it also carries the risk of a pyometra, development of stress incontinence due to alteration of the urethrovesical angle and any existing enterocoele will not be corrected by this operation. ${ }^{2}$

Careful trans-vaginal ultrasound examination and cervical cytology can be made mandatory before opting for colpocleisis. ${ }^{9}$

Study conducted by Winkelman et al concluded that over the time the patients may report with bowel and bladder symptoms as a result of the surgery. Hence a detailed consent and expectation setting is required for women before considering colpocleisis. ${ }^{8}$

Park JY et al have quoted that occult SUI may occur in about $9.9 \%$ to $68 \%$ women but they will be mild, transient and self resolving. ${ }^{9}$

Among the elderly women who visit the general physician with signs and symptoms of renal failure an attempt has to be made to perform the gynaecological examination for pelvic organ prolapse. ${ }^{10}$

\section{CONCLUSION}

Colpocleisis should be considered as one of the surgical options for advanced pelvic organ prolapse in elderly patients who do not intend to preserve vaginal function for sexual function. All urogynecologists should become familiar with this surgical procedure and always offer it as one surgical option for elderly patients with advanced pelvic organ prolapse.
Funding: No funding sources

Conflict of interest: None declared

Ethical approval: Not required

\section{REFERENCES}

1. Ng SC, Chen GD. Obliterative Le Fortcolpocleisis for pelvic organ prolapse in elderly women aged 70 years and over. Taiwanese J Obstet Gynecol. 2016;55:68-71.

2. Formosa M. Le Fort Colpocleisis:re-visited and reproposed. Malta Med J. 2009;21(3):36-7.

3. Miyagil A, Inaguma Y, Tokoyoda T, Nakajima T, Sezaki R, Matsukawa T. A case of renal dysfunction caused by pelvic organ prolapse. CEN Case Rep. 2017;6:125-8.

4. Brettauer J, Rubin IC. Hydroureter and hydronephrosis: a frequent secondary finding in cases of prolapse of the uterus and bladder. Am $\mathbf{J}$ Obstet Gynecol. 1923;6:696-708.

5. Kurt S, Guler T, Canda MT, Demirtas Ö, Tasyurt A. Treatment of uterine prolapse with bilateral hydronephrosis in a young nulliparous woman; a new minimally invasive extraperitoneal technique. Eur Rev Med Pharmacol Sci. 2014;18(11):1657-60.

6. Lamers BH, Broekman BM, Milani AL. Pessary treatment for pelvic organ prolapse and healthrelated quality of life: a review. Int Urogynecol J. 2011;22(6):637-44.

7. Jelovsek JE, Maher C, Barber MD. Pelvic organ prolapse. Lancet. 2007;369(9566):1027-38.

8. Winkelman WD. Long term pelvic floor symptoms, Recurrence, Satisfaction and regret following colpocleisis. Female Pelvic Med Reconst Surg. 2018.

9. Park JY, Han SJ, Kim JH, Chun KC, Lee TS. Le fort partial Colpocleisis as an effective treatment option in elderly women. TJOG. 2019;58:206-11.

10. Sato S, Nishida M. Renal Failure due to severe pelviic organ prolapsed. J General Family Med. 2016;17(3):249-51.

Cite this article as: Rohini E, Hiremath $\mathrm{PB}$, Sanyal U, Indu NR, Ghosh S, Hiremath R. Obliterative le fort's colpocleisis for acute kidney injury caused by pelvic organ prolapse in elderly woman. Int J Reprod Contracept Obstet Gynecol 2019;8:4083-5. 